\title{
Polysèmes
}

Revue d'études intertextuelles et intermédiales

\section{Visible/invisible/visuel : spectralité et hantologie dans The Gateless Barrier de Lucas Malet}

Visible, invisible, visual: spectrality and hauntology in Lucas Malet's

The Gateless Barrier

\section{Catherine Delyfer}

\section{OpenEdition}

Journals

Édition électronique

URL : http://journals.openedition.org/polysemes/315

DOI : 10.4000/polysemes.315

ISSN : 2496-4212

Éditeur

SAIT

Référence électronique

Catherine Delyfer, «Visible/invisible/visuel : spectralité et hantologie dans The Gateless Barrier de Lucas Malet », Polysèmes [En ligne], 13 | 2015, mis en ligne le 14 octobre 2015, consulté le 20 avril 2019. URL : http://journals.openedition.org/polysemes/315 ; DOI : 10.4000/polysemes.315

Ce document a été généré automatiquement le 20 avril 2019

Polysèmes 


\section{Visible/invisible/visuel : spectralité et hantologie dans The Gateless Barrier de Lucas Malet}

Visible, invisible, visual: spectrality and hauntology in Lucas Malet's

The Gateless Barrier

Catherine Delyfer

1 The Gateless Barrier est un roman anglais de 1900 qui accorde une place centrale à l'imagination, aux passions, à l'insondable, l'impalpable, l'inexplicable. En choisissant pour héroïne une jeune morte-vivante, la charmante Agnes Rivers, il déroge aux lois du roman réaliste pour mieux semer le désordre dans le récit conventionnel, le politiser autour de la question du genre, et même jeter le trouble dans l'historiographie littéraire, en ramenant au centre de ses préoccupations les questions de hantise, de survivance, d'héritage, de tradition, de responsabilité, de dette et de justice. Comme la fable d'Oscar Wilde The Fisherman and His Soul, ou encore The Picture of Dorian Gray, ce roman de «Lucas Malet » (Mary St Leger Kingsley Harrison, 1852-1931) mêle la fascination fin de siècle pour les rites catholiques, l'occulte, le surnaturel, l'ésotérique et le spiritisme avec les ingrédients classiques du roman gothique, qui repose sur la dialectique de l'impalpable rendu visible.

2 Dans ce texte, le genre gothique est réactualisé pour donner corps à certaines angoisses culturelles propres à la fin du dix-neuvième siècle britannique, puis les dissiper, car ce n'est pas un roman sombre, mais au contraire plein d'optimisme, de malice et de légèreté. En effet, le personnage principal, Laurence Rivers, écrivain à la dérive, y sera initié aux mystères du « Veil of Isis " ${ }^{1}$, c'est-à-dire aux mystères théosophiques de l'au-delà et de la réincarnation chers à madame Blavatsky, afin de pouvoir faire face à son destin de manière plus constructive et plus résolue. Notons que cette image du voile est une image paradoxale en cela que, tout comme la notion de transparence qu'elle convoque, elle est tendue entre deux extrêmes, évoquant à la fois l'ouverture et la fermeture, le matériel et l'immatériel, une présence et une absence, une visibilité maximale (celle de l'objet révélé 
par transparence) et une quasi-parfaite invisibilité (celle du voile lui-même). Du reste, cette notion de voile se marie parfaitement avec la figure du spectre, cette "absenceprésente $»^{2}$ qui est être et non-être, corps et âme, chair et esprit.

3 En guise de préface à son sixième roman, Lucas Malet cite un passage tiré du texte de Lafcadio Hearn, Exotics and Retrospectives (1898). Cet extrait joue un rôle parergonique évident: il constitue le premier des nombreux seuils, tangibles ou intangibles, que le lecteur sera invité à franchir. Il sert de point de départ pour aborder le récit qui suit sous l'angle voulu par l'auteur, un angle d'approche qui fait subir au lecteur une certaine désorientation, une certaine dé-territorialisation. Non sans humour, cette mise en condition exige en effet que le lecteur accepte d'envisager ce roman comme un Mun-MonKwan, le chinois pour "gateless barrier ", une expression, explique Lafcadio Hearn dans la citation, qui désigne les livres d'étude privilégiés des écoles dhyâna bouddhistes, des ouvrages supposés permettre de s'exercer à une forme de méditation menant à l'état de dhyâna, c'est-à-dire un état de conscience supérieur, transcendant, censé être également un état d'énergie et de joie intenses. En conséquence, la préface invite le lecteur à appréhender ce roman non de manière dénotative, mais de manière connotative et contemplative, comme un texte «not explanatory. [It] only suggest[s] »3. À une époque où on attendait des écrivains femmes qu'elles se limitent au genre réaliste, et plus exactement au récit d'événements vraisemblables relevant de la sphère privée, cet avertissement n'était pas superflu. Mais il faut croire qu'il fut négligé par les critiques contemporains qui reprochèrent précisément à cette œuvre ses événements peu plausibles ${ }^{4}$. The Gateless Barrier est en réalité gouverné par des impératifs et une logique tout autres, propres au genre $\mathrm{du}$ « feminine gothic $»^{5}$ dans la tradition radclifféenne.

Enfin, la courte préface du roman est aussi l'occasion de poser une certaine méfiance à l'égard du matériau linguistique lui-même: comme les écrits dhyâna, auxquels il est implicitement comparé, ce texte « represents human effort to reach [...] zones of thought beyond the range of verbal expression ». "Questions are put, but the student must think out the answers for himself. He must think them out, but not write them », car " any thought narrowed into utterance loses all Dhyâna quality $»^{6}$. Ici c'est donc le texte luimême qui aspire à devenir fantôme et à hanter le lecteur par le questionnement qu'il suscite. Quel est alors ce texte qui refuse d'en être un et qui veut évoquer l'indicible en faisant rayonner une pensée affranchie de l'entrave des mots? Comment s'y prend-il pour dépasser sa propre chair verbale? Quelle est donc cette forme d'expression littéraire légère, libre et efficace qui tente d'assurer au texte son invisibilité, sa transparence en quelque sorte?

\section{Transparence et fantômalité}

5 The Gateless Barrier raconte l'histoire de Laurence Rivers, un jeune écrivain anglais vivant à New York qui est soudain rappelé en Angleterre, au chevet de son grand-oncle mourant. La vie outre-Atlantique de Laurence, dominée par sa fringante épouse américaine, une «new woman » riche, indépendante et pleine d'assurance, forme le récit enchâssant de l'aventure gothique qui va suivre, la traversée de l'Atlantique servant de zone de passage entre les deux récits, tout en suggérant aussi des ponts entre ces deux traditions littéraires que sont le genre gothique et le genre du « New Woman novel». Seul héritier de la grande demeure ancestrale et des collections qu'elle abrite, Laurence s'empresse de regagner l'Angleterre. Il tombe immédiatement sous le charme de ce domaine qu'il a 
connu enfant et qu'il a plaisir à explorer de nouveau, entre deux entrevues avec son vieil oncle qui s'éteint peu à peu. Le roman se concentre sur la demeure, espace ô combien symbolique, dont les peintures, les objets de décoration, les livres et le mobilier reflètent la personnalité des divers membres de la famille qui l'ont occupée au fil du temps et incarne ainsi, d'une certaine manière, toute l'histoire de la culture et de la littérature britannique. Comme le fait remarquer Talia Schaffer, en héritant de cette propriété, Laurence hérite aussi en d'autres termes de ce que F.R. Leavis nommerait plus tard « the great tradition $»^{7}$.

6 Montagu Rivers, le grand-oncle mourant, est homme de lettres et collectionneur d'œuvres d'art rares et exotiques. C'est un érudit hautain, acariâtre et cynique qui ne se préoccupe guère du vulgaire, l'immense savoir qu'il a accumulé n'étant pas destiné à être partagé, mais seulement à satisfaire sa mégalomanie personnelle. Sa bibliothèque révèle qu'il s'intéresse aux œuvres littéraires, scientifiques et philosophiques les plus subversives de son temps, et que les Rivers en général ont toujours eu un penchant pour les textes dangereux - ceux de Thomas Paine et de Jean-Jacques Rousseau, par exemple, que l'un de leurs ancêtres lisait avec ferveur à la fin du dix-huitième siècle. Dandy jusque dans son lit de mort, Montagu Rivers continue de porter son bonnet de velours violet, sa robe de chambre doublée de fourrure et ses bagues orientales trop lourdes pour ses mains décharnées, suivant là un code vestimentaire rendu célèbre par Oscar Wilde. Depuis des années, Rivers vit retiré dans sa grande et sombre demeure tapissée de lambris de chêne et de rideaux de damas bleu : les fenêtres y sont toujours maintenues fermées, l'éclairage y est purement artificiel et la chaleur de l'air y semble d'autant plus suffocante que l'atmosphère est chargée de parfums musqués capiteux. L'intérieur est agrémenté, en outre, de magnifiques bouquets d'orchidées et de fleurs vénéneuses, d'une collection de bustes immortalisant les empereurs romains les moins recommandables, de statues d'ébènes représentant des sphinx lubriques et de tapisseries mettant en scène les épisodes les plus libidineux de la littérature classique. Bref, Montagu Rivers est un représentant aisément reconnaissable de l'Esthétisme décadent, comme Gilbert Osmond ou Jean Des Esseintes avant lui. Sa misogynie extrême vient parachever le portrait de ce dandy fin de siècle: aucune visiteuse n'est admise à Stoke Rivers et le vieil homme s'entoure de valets et de cuisiniers exclusivement masculins, car pour lui comme pour Baudelaire, la femme n'est qu'une machine à procréer vile, bestiale et hystérique : aux antipodes du dandy, la femme est "naturelle, c'est-à-dire abominable $»^{8}$. Dans ces conditions, il n'est guère surprenant que le retour du refoulé prenne dans ce roman la forme d'une présence fantômale (et symptômale) féminine. Du reste, à l'approche de la mort, le vieux dandy est tourmenté par la conscience qu'il a d'avoir délibérément ignoré cette forme d'humanité lors de son séjour terrestre, et tel Hamlet méditant sur la mort d'Ophélie et l'apparition de son père défunt, Montagu Rivers rumine ses sombres pensées en tenant entre les mains un beau crâne de cristal' 9

7 Il est vrai qu'au cœur de la demeure patriarcale, existe une pièce secrète dans laquelle personne n'ose pénétrer, et dont l'entrée est barrée par une splendide tapisserie doublée de cuir. Matérialisant la proximité troublante du sacré et du profane, cette tenture rappelle au protagoniste le genre de rideau utilisé devant l'entrée des églises romanes, tandis qu'il reconnaît dans le motif qui y est brodé une scène inspirée du viol de Lucrèce. L'étoffe, en apparence pesante et rigide, est en réalité souple et soyeuse au toucher, et constitue un seuil paradoxal qui est à la fois invitation au franchissement et barrage, ouverture et fermeture, dedans et dehors. Nous sommes, pour parler comme Georges 
Didi-Huberman, "entre un devant et un dedans $»^{10}$. De fait, lorsque le jeune héritier Laurence Rivers s'aventure derrière l'étrange rideau pour la première fois, il est tout d'abord terrifié par la densité de l'air qui semble soudain s'opacifier et s'épaissir pour acquérir une résistance, une solidité oppressante qui l'étouffe : «He fumbled blindly in the dark, an almost childish sense of agitation upon him. He felt as in dreams, when the place to be traversed grows more contracted, walls closing down and in on every hand, while the means of exit becomes more maddeningly impossible of discovery. To his surprise, he turned faint and broke into a sweat ${ }^{11}$. Or cet affolement passager qui saisit le personnage masculin correspond clairement au sentiment d'inquiétante étrangeté freudien, que Didi-Huberman analyse comme une expérience de désorientation du regard dans laquelle

[...] nous ne savons plus exactement ce qui est devant nous et ce qui ne l'est pas, ou bien si le lieu vers où nous nous dirigeons n'est pas déjà ce dedans quoi nous serions depuis toujours prisonniers. [...] Or, c'est en dernière limite devant le sexe féminin, nous dit Freud, que les « hommes névrosés » - c'est-à-dire les hommes en général éprouvent le plus cette désorientation de l'Unheimliche : c'est lorsque s'ouvre devant eux ce lieu étrange, si étrange en vérité parce qu'il impose ce retour au "chez soi » (das Heimische) perdu, au seuil passé de toute naissance. La référence métapsychologique à l'angoisse de castration se complète donc ici d'une référence au «fantasme du ventre maternel » [...]. [L]a désorientation naît d'une limite qui s'efface ou vacille, par exemple entre la réalité matérielle et la réalité psychique. ${ }^{12}$

Il n'est donc guère surprenant que lorsque Laurence parvient enfin à saisir la poignée et que la porte s'ouvre, il se retrouve dans un espace saturé de féminité. Loin d'être menaçant, ce lieu auquel il accède est exquis et décoré dans le style gai, coloré, sobre et élégant du dix-huitième siècle. Cette pièce, nommée "the yellow drawing room », en référence à la nouvelle gothique féministe de Charlotte Perkins Gilman (publiée en 1892), est habitée par le fantôme d'Agnes Rivers, et offre un contraste saisissant avec le reste de la maison, puisqu'elle jouit d'une atmosphère pure, fraîche et vivifiante, donne sur un joli jardin à l'italienne et regorge de témoignages de la créativité féminine. La limite qu'opposait le rideau brodé de motifs misogynes s'efface, tandis que s'ouvre un nouvel horizon de visibilité et d'entendement.

9 Au fur et à mesure de ses visites dans le salon jaune, Laurence observe avec fascination, puis amour, l'apparition vaporeuse de la femme fantôme dont les mouvements agitent l'air aussi imperceptiblement que les battements d'ailes d'un papillon. Coiffée selon la mode du tout début du dix-neuvième siècle, vêtue d'une robe fluide à taille haute, ses épaules presque invisibles sous le voile de mousseline qui les recouvre, Agnes apparaît et disparaît mystérieusement derrière l'écritoire placé dans un coin de la pièce, un écritoire qu'elle semble chercher désespérément à ouvrir :

The young lady's hands moved over the rounded cover of the escritoire as though endeavouring to open it [...] She touched, yet seemed unable firmly to grasp, the gilded handles of the escritoire again and again [...] with a baffled, despairing gesture, and then moved away across the room. She appeared to flit rather than walk, so light and silent were her steps, bird-like in their swift and dainty dance [...] The flitting figure now before him, notwithstanding the beguiling loveliness of its motions, struck him as penetrate with the sorrow of failure [...] She wandered to and fro, regardless or unconscious of his presence, searching-searching-as it seemed [...] Then reluctantly, as in obedience to some unwelcome summons, she passed at the back of [the outstanding, painted satinwood escritoire], and the young man found himself alone. ${ }^{13}$ 
Cet écritoire en bois précieux que le joli fantôme venu de l'époque georgienne ne peut plus ouvrir, puisque ses mains transparentes et désincarnées sont désormais impuissantes à saisir les choses, c'est en réalité l'histoire littéraire et intellectuelle ellemême, dont les femmes de la fin de l'époque victorienne sont de plus en plus exclues. De fait, des critiques telles que Lyn Pykett, Antonia Losano, Talia Schaffer ou Ann Ardis ont récemment montré que le tournant du siècle avait été particulièrement impitoyable à l'endroit des femmes artistes et écrivains, faisant régresser leur statut en dénigrant systématiquement leur contribution à l'histoire culturelle de leur temps, pour finir par occulter totalement leur rôle dans le bouleversement des formes et des sujets littéraires et l'avènement du Modernisme, entre 1880 et 1914. L'image de l'écritoire comme métaphore de l'écriture féminine est encore développée et soulignée avec insistance, lorsque l'on apprend plus tard qu'au lieu de recevoir les funérailles chrétiennes qui lui étaient dues, le corps d'Agnes a été confisqué par un cousin amoureux et jaloux, et qu'à sa place, pour alourdir le cercueil, se trouvent des livres ${ }^{14}:$ l'écriture féminine est bel et bien enterrée. La transparence du fantôme thématise alors le défaut de mémoire, car l'absence du corps d'Agnes dans le tombeau familial des Rivers signifie qu'aucune forme de culte rendu en l'honneur de la défunte n'est possible. Le corps transparent sert ainsi de figure pour représenter le ressentiment légitime des dominés, des vaincus et des oubliés de la culture, condamnés à une existence spectrale dans leur propre société.

\section{Transparence et hantise}

11 Toutefois, le corps fantômal est plus complexe que cela, plus puissant aussi, au même titre d'ailleurs que ces autres corps transparents ou à peine visibles, tels que l'air, la fumée ou la poussière, étudiés par Georges Didi-Huberman dans son livre sur l'art de Claudio Parmiggiani, Génie du non-lieu. En effet, même si le fantôme peine à devenir visible, même s'il y a toujours, selon Derrida, « du disparu dans l'apparition même comme réapparition du disparu $»^{15}$, le spectre reste toutefois, et peut-être avant tout, une incorporation, une incarnation, un « devenir-corps ", pour parler comme Derrida, c'est-à-dire une certaine forme phénoménale et charnelle de l'esprit. En cela il se différencie du simulacre. Car, écrit encore Derrida, "la chair, la phénoménalité, voilà ce qui donne à l'esprit son apparition spectrale $»^{16}$. Dans The Gateless Barrier, nous allons voir que l'incorporation paradoxale du spectre est signifiée dans toute sa présence, son effectivité potentielle, sa puissance et sa force de déploiement auratique, lors d'une scène de trans-substantiation inspirée du rituel catholique.

12 Au fur et à mesure que ses forces le quittent, le vieil oncle mourant n'est plus que l'ombre de lui-même; il devient de plus en plus «transparent ${ }^{17}$ et son visage, nous dit-on, est tellement pâle qu'il semble disparaître contre le fond blanc de l'oreiller. Inversement, la belle Agnes semble gagner en matérialité, en phénoménalité, et même en couleur, surtout après le décès de Montagu Rivers, au milieu du roman. Grâce à l'amour du jeune écrivain héritier Laurence Rivers, qui tente ardemment de communiquer avec elle et d'entendre les paroles muettes qu'elle semble articuler, Agnes retrouve la voix, ou plus exactement sa voix redevient audible. La visibilité renforcée de la belle apparition, ainsi que son audibilité retrouvée participent ainsi d'un nouveau "partage du sensible», au sens de Jacques Rancière, c'est-à-dire d'un événement esthétique qui est aussi une reconfiguration d'ordre politique dressant un nouvel état des forces. Car quand le récit littéraire façonne de nouvelles auditions, de nouvelles écoutes, de nouvelles visibilités, de 
nouvelles sensorialités tout en ruinant d'anciennes hiérarchies, il aide alors, pour Rancière, à conceptualiser comme des réalités sensibles des libertés et des égalités jusqu'alors impensables. Ici, c'est la vie d'Agnes, son œuvre, puis enfin son esprit, qui sont ramenés à la conscience collective et bousculent ainsi l'histoire de son temps.

Tout d'abord, l'écritoire scellé qui renferme la correspondance (l'œuvre) d'Agnes est enfin déverrouillé, grâce à Laurence, à l'aide d'une «toute petite clé ", la treizième du trousseau - un peu comme dans le conte de Barbe-Bleu, où la clé du placard interdit est l'instrument de la cruauté patriarcale en même temps qu'elle sera finalement le moyen de s'y soustraire définitivement. Mais enhardi par ces succès et brûlant de désir, Laurence tente alors le tout pour le tout afin de ramener complètement Agnes à la vie terrestre et pouvoir s'unir à elle. Or cette tentative orphique est bien entendu vouée à l'échec : tout comme Orphée renvoie fatalement Eurydice dans le royaume des morts par son désir insoutenable de se retourner pour la posséder du regard, Laurence perd son fantôme en cherchant à la faire sienne. En effet, se croyant l'égal de Dieu, Laurence décide de célébrer, à sa façon, le sacrement de l'Eucharistie, en pressant Agnes de manger du pain et des raisins. Mais, comme le fait remarquer Schaffer, à l'instant crucial, en bonne chrétienne, le « ghost » refuse de jouer le rôle du « holy ghost » et la trans-substantiation, c'est-à-dire la conversion du pain en chair et du raisin en sang, ne peut avoir lieu. Renonçant à ces amours supranormales, la spectrale Agnes quitte alors Laurence et le renvoie en Amérique, en marge du récit enchâssé, vers son épouse et vers les conflits du monde réel moderne. Quelque temps plus tard, un incendie inexpliqué détruit entièrement le salon jaune, et l'on découvre alors, comme dans « The Black Cat » d'Edgar Allan Poe, les restes calcinés d'Agnes Rivers, dont le corps avait été emmuré au début du siècle dans un recoin derrière l'écritoire par un cousin jaloux ressemblant trait pour trait au défunt Montagu Rivers. Cette partie du récit se conclut néanmoins sur une note optimiste car, en brûlant, le corps d'Agnes libère son esprit, à l'instar de ce qui se produit dans la nouvelle de Vernon Lee, «The Doll» (1896). Qui plus est, après avoir donné sa place à la défunte dans le mausolée familial, Laurence aménage son bureau d'écrivain dans le salon jaune rénové où, entouré des ouvrages légués par son grand-oncle et habité par l'esprit d'Agnes, il sera à même de cultiver son double héritage (féminin et masculin) et de produire une littérature et une histoire intellectuelle moins hostiles aux femmes, qui soit le fruit des contributions des unes et des autres. Il n'est donc pas surprenant que le roman finisse en précisant que l'entourage de Laurence trouve l'écrivain de plus en plus «queer» - une façon pour Lucas Malet d'appeler à une hybridation culturelle qui saurait dépasser les problématiques du genre, c'est-à-dire à une véritable histoire « queer » de la culture et de la littérature.

De la maison hantée à la maison brûlée, suivant un parcours inverse à l'analyse de DidiHuberman dans Génie du non-lieu, Lucas Malet médite ainsi à sa façon sur la transparence qui n'est pas le néant, mais qui est la conscience d'une absence, c'est-à-dire le deuil et la mémoire d'une présence. C'est pourquoi il n'est peut-être pas exagéré de dire que ce que la romancière propose, dans ce petit texte par ailleurs si modeste, c'est une " hantologie " à la Derrida, c'est-à-dire une pensée de l'être, et de l'être écrivain tout particulièrement, qui soit plus puissante et plus large qu'une ontologie, en cela qu'elle embrasserait non seulement l'être, mais aussi le non-être, ou le presque-être, que constituent des générations de spectres, afin d'en honorer les restes, de dire leur absence et de les rendre ainsi présents. C'est ainsi que l'on peut comprendre la révélation finale du protagoniste masculin: 
The moon had risen, but its mild light was often obscured by softly-moving floats of thin, opalescent vapour. These crossed its face in apparently endless procession, herded up from southward and the narrow Channel Sea. Laurence watched them, at first almost unconsciously, his mind occupied with other, and, to himself, more immediate and vital interests. But at length their slow and stately progress began to work upon his imagination, and insinuate itself into the very substance and foundation of his thought. He began to see in them a procession of the souls of all those generations of men and women, whose efforts and emotions, power of intellect, fiercely pursued ambitions, passionate devotions, passionate revolts, had gone to generate his own constitution, mental and physical, and determine his ultimate fate. And so he came to regard them with a sustained and deepening attention, since their aspect seemed pregnant with suggestion of admonition, of encouragement, of warning, or restraint. Once again he decided to keep vigil in this house, to watch with the unnumbered and unrecorded dead whose offspring and inheritor he was. ${ }^{18}$

\section{Transparence et visualité}

15 Comme on peut le constater à la lecture de ce passage, l'épiphanie de Laurence Rivers est communiquée au lecteur par une forte sollicitation de son œil intérieur: "sight » et « insight» semblent ici intimement mêlés, puisque c'est ce que Laurence voit sans le regarder vraiment qui interrompt le fil de sa pensée et impulse une bifurcation interprétative imprévue qui lui fait l'effet d'une révélation fulgurante et lui permet de reconfigurer sa compréhension du monde. Dans le face-à-face de Laurence avec la lune, le visage de l'histoire semble se redessiner. Cette nouvelle "visagéité ", au sens de Deleuze et de Lévinas, est elle-même préparée et appelée par la présence dans le roman de deux autres visages obsédants, ceux des portraits qui sont cachés dans l'un des tiroirs de l'écritoire - cet écritoire auquel je reviens avant de conclure.

16 J'ai montré dans mes travaux antérieurs sur la poétique visuelle de Lucas Malet que l'écriture maletienne puise sa force d'intervention et de liberté dans les interstices qu'elle creuse entre textuel et pictural ${ }^{19}$. En étudiant les nombreux tableaux, petits ou grands, accrochés aux murs d'autres romans de Malet, j'ai observé que le recours au visuel est toujours, chez cette peintre contrariée, trace et source d'une friction, d'une dissidence, d'une résistance, d'une différance productrice et féconde. En outre, l'image maletienne est en général authentiquement "dialectique", dans le sens donné à ce terme par Georges Didi-Huberman : à la fois présence et représentation, l'image chez Malet effectue en effet un télescopage, une rencontre fulgurante entre passé, présent et avenir ; bref, elle constitue un anachronisme, une crise de temps, une expérience intérieure. The Gateless Barrier ne fait pas exception, comme on va le voir.

Lorsque Laurence parvient à ouvrir l'écritoire et qu'il découvre les lettres d'Agnes, toutes datées entre 1802 et 1805, il trouve aussi une multitude de petits souvenirs, keepsakes conservés avec amour par la jeune femme. Mèche de cheveux, boucle de chaussure, ruban, petits coquillages, bracelets tressés: autant d'objets ordinaires transmués en trésors ou en reliques dans l'esprit de celle pour qui leur valeur est entièrement sentimentale. Avec d'infinies précautions, Laurence soulève l'un de ces mémentos parfaitement préservés, un petit bouquet de fleurs séchées, qui brusquement s'effritent et retombent en poussière. Cette métaphore d'un temps pulvérisé, vaporisé, souffléz ${ }^{20}$ devenu transparent, fait alors bifurquer le récit, comme si cette poussière de fleurs séchées faisait basculer Laurence et le lecteur dans une autre temporalité, qui n'est ni narrative ni chronologique mais à la dérive, atopique, intempestive - le temps de l'image, 
le temps du visuel. Car, l'instant d'après, Laurence découvre dans l'écritoire « wrapped, face to face, in a lace and lawn handkerchief, two very exquisite miniatures by Cosway [... ], the radiant, pictured faces, the two graceful, young heads turned slightly towards each other as in mutual tenderness and sympathy $»^{21}$. Ces délicates peintures du célèbre miniaturiste anglais le frappent de stupeur. En effet,

[...] one miniature represented his fairy-lady [...]. And the other miniature, by the same hand, showing the same delightful sympathy of artist with his subject, touched by the same poetic insight and grace was a portrait of whom? Well, of himself-himself, Laurence Rivers [...]. He had been a sailor apparently, for he wore the dark-blue, naval uniform of the early years of the century, his brown hair being tied back into a queue. But for these details the resemblance to himself was absolute. And then, suddenly, [he felt] a sense of faintness as though his identity were slipping away from him, and his hold on actuality loosening as he imagined it might loosen in the moments immediately preceding death [...]. ${ }^{22}$

Plus que la rencontre avec le fantôme de son arrière-cousine Agnes, c'est ce face-à-face avec le portrait de son propre ancêtre, sosie et homonyme, le Laurence Rivers tombé à Trafalgar avant d'avoir pu se marier avec la douce Agnes, qui pétrifie le jeune homme. En effet, ce portrait fait voler en éclats tout sentiment d'identité chez l'actuel Laurence qui a soudain l'impression d'être la pâle copie d'un original insoupçonné plus illustre, dont il reproduit le physique, les émotions, les paroles, les amours, les choix, les actes. La miniature vient ainsi rompre cette spontanéité, cette illusion de transparence de soi à soi qui sert de fondement au sentiment identitaire, et elle coupe Laurence de lui-même. L'image du visage de l'autre Laurence fait effraction dans son être, le déchire de luimême, ouvre une scission en lui, lui vole son intimité et le rend invisible. Mais cette cassure est fondatrice et, si l'on en croit Emmanuel Lévinas, essentielle dans tout parcours éthique. Car, selon le philosophe, c'est l'altérité même du visage de l'autre qui nous tire de nous-même. Dans le roman de Malet, c'est l'altérité radicale (car rationnellement impossible) du visage de l'autre Laurence, qui décentre et déloge le Laurence actuel, qui constitue l'étape préalable indispensable à sa position éthique d'écrivain à la fin du roman. Davantage encore que la rencontre avec le spectre d'Agnes, c'est donc la découverte de sa propre ré-incarnation, du fantôme qui l'habite (et/ou qu'il habite), qui représente pour Laurence le tournant majeur du récit, un moment proprement « spectral », ce moment derridien,

[...] qui n'appartient plus au temps, si l'on entend sous ce nom l'enchaînement des présents modalisés (présent passé, présent actuel : «maintenant », présent futur). Nous questionnons à cet instant, nous nous interrogeons sur cet instant qui n'est pas docile au temps, du moins à ce que nous appelons ainsi. Furtive et intempestive, l'apparition du spectre n'appartient pas à ce temps-là, elle ne donne pas le temps, pas celui-là. ${ }^{23}$

De manière assez caractéristique, Lucas Malet représente ce basculement vers le spectral comme un moment ekphrastique, un moment de visualité pure où l'image picturale (la miniature) se lève dans le texte, et où la relève du texte par l'image fait événement. Cet événement, ce surgissement, n'est pas sans rappeler la traversée du rideau et de la porte menant au salon jaune. Là encore, les analyses fines de Didi-Huberman sont d'une grande aide pour comprendre ce qui se joue dans ce moment ekphrastique conceptualisé comme un seuil (un de plus). Dans son chapitre sur le motif de la porte, «L'interminable seuil du regard ", Didi-Huberman propose en effet que

Regarder, ce serait prendre acte que l'image est structurée comme un devant-dedans

: inaccessible et imposant sa distance, si proche soit-elle - car c'est la distance d'un 
contact suspendu, d'un impossible rapport de chair à chair. Cela veut juste dire - et d'une façon qui n'est pas seulement allégorique - que l'image est structurée comme un seuil. Un cadre de porte ouverte, par exemple. Une trame singulière d'espace ouvert et clos en même temps. Une brèche dans un mur, ou une déchirure, mais œuvrée, construite, comme s'il fallait un architecte ou un sculpteur pour donner forme à nos blessures les plus intimes. Pour donner, à la scission de ce qui nous regarde dans ce que nous voyons, une espèce de géométrie fondamentale. ${ }^{24}$

Pour conclure, et pour répondre succinctement à la dernière question que je posai en introduction (« quelle est donc cette forme d'expression littéraire qui tente d'assurer au texte sa transparence?»), je dirai que c'est la qualité icono-textuelle de ce roman qui ménage des zones de transparence, des trouées intempestives dans le texte. Comme souvent chez Malet, le récit et l'écriture sont mus par et vers le pictural, et le lecteur est ému par la conscience soudaine d'une zone d'intermittence qui fait comme une percée intérieure ou une éclaircie visuelle à un moment de crise. Pour la romancière-peintre comme pour Didi-Huberman, "l'image mieux que toute autre chose, probablement, manifeste cet état de survivance qui n'appartient ni à la vie tout à fait, ni à la mort tout à fait, mais à un genre d'état aussi paradoxal que celui des spectres qui, sans relâche, mettent du dedans notre mémoire en mouvement $»^{25}$. C'est d'ailleurs cet attachement aux pouvoirs de l'image picturale en texte, à la puissance du visuel (qui à la fois rend visible et désagrège, déborde le visible), qui fait de Lucas Malet une grande romancière dans la tradition littéraire de l'Esthétisme, et non une simple militante en faveur des droits des femmes.

\section{BIBLIOGRAPHIE}

Baudelaire, Charles. Journaux intimes : Fusées, Mon Cœur mis à nu. Paris : G. Crès, 1887.

Derrida, Jacques. Spectres de Marx. Paris : Galilée, 1993.

Didi-Huberman, Georges. Devant l'image. Questions posées aux fins d'une histoire de l'art. Paris :

Éditions de Minuit, 1990.

Didi-Huberman, Georges. Ce que nous voyons, ce qui nous regarde. Paris : Éditions de Minuit, 1992.

Didi-Huberman, Georges. Devant le temps. Histoire de l'art et anachronisme des images. Paris : Éditions de Minuit, 2000.

Didi-Huberman, Georges. Génie du non-lieu. Air, poussière, empreinte, hantise. Paris : Éditions de Minuit, 2001.

Lee, Vernon. «The Doll » (1899). For Maurice: Five Unlikely Stories. London: John Lane, 1927, 207-221.

Lévinas, Emmanuel. Totalité et infini (1961). Paris : Poche, 1990.

Lundberg, Patricia Lorimer. "An Inward Necessity »: the Writer's Life of Lucas Malet. New York: Peter Lang, 2003.

Malet, Lucas. The Gateless Barrier. New York: Dodd, Mead \& Company, 1900. 
Poe, Edgar Allan. « The Black Cat » (1843). Complete Short Stories and Poems of Edgar Allan Poe. New York: Doubleday, 1984, 63-69.

Rancière, Jacques. Le Partage du sensible : esthétique et politique. Paris : La Fabrique Éditions, 2000.

Schaffer, Talia. « Some Chapter of Some Other Story: Henry James, Lucas Malet, and the Real Past of The Sense of the Past ». The Henry James Review 17.2 (1996): 109-128.

Williams, Ann. Art of Darkness: A Poetics of Gothic. Chicago: Chicago UP, 1995.

\section{NOTES}

1. Lucas Malet, The Gateless Barrier, New York: Dodd, Mead \& Company, 1900, 180.

2. L'expression est de Georges Didi-Huberman, dans Génie du non-lieu (Paris : Éditions de Minuit, 2001), 126.

3. Malet, op. cit., v.

4. Pour la réception de The Gateless Barrier, se reporter à la biographie de l'auteur par Patricia Lorimer Lundberg, "An Inward Necessity »: the Writer's Life of Lucas Malet, New York: Peter Lang, 2003.

5. La distinction entre genre gothique masculin et genre gothique féminin est explicitée par Ann Williams, dans son classique Art of Darkness: A Poetics of Gothic (Chicago UP, 1995).

6. Malet, op. cit., v-vi.

7. Talia Schaffer, «Some Chapter of Some Other Story: Henry James, Lucas Malet, and the Real Past of The Sense of the Past ", The Henry James Review 17.2 (1996): 110.

8. Charles Baudelaire, Journaux intimes: Fusées, Mon Cour mis à nu, Paris: G. Crès, 1887, 48. Italiques dans le texte original.

9. Malet, op. cit., 86-89.

10. Georges Didi-Huberman, Ce que nous voyons, ce qui nous regarde, Paris : Éditions de Minuit, 1992, 185.

11. Malet, op. cit., 28.

12. Didi-Huberman, Ce que nous voyons, ce qui nous regarde, op. cit., 183-184.

13. Malet, op. cit., 50-55.

14. Ibid., 170.

15. Jacques Derrida, Spectres de Marx, Paris : Galilée, 1993, 25.

16. Ibid.

17. Malet, op. cit., 11, 14, 80, 87.

18. Ibid., 345, mes italiques.

19. Voir mon livre, Art and Womanhood in Fin-de-Siècle Writing: the Fiction of Lucas Malet, 1880-1931 (London: Pickering and Chatto, 2011).

20. J'emprunte l'image d'un temps "soufflé " à Didi-Huberman qui consacre un chapitre aux traces et aux espaces « soufflés » dans Génie du non-lieu (Paris : Éditions de Minuit, 2001).

21. Malet, op. cit., 147.

22. Ibid., 147-149.

23. Derrida, Spectres de Marx, Paris : Galilée, 1993, 17.

24. Didi-Huberman, Ce que nous voyons, ce qui nous regarde, op. cit., 192-193.

25. Didi-Huberman, Génie du non-lieu, op. cit., 2001, 16. 


\section{RÉSUMÉS}

The Gateless Barrier (1900) est un roman qui accorde une place centrale au visuel tel que l'entend Georges Didi-Huberman, c'est-à-dire à l'insondable, l'impalpable, l'inexplicable de l'image. En choisissant pour héroïne une jeune morte-vivante, la charmante Agnes Rivers, Malet déroge aux lois du roman réaliste comme à celles du roman esthétique décadent pour mieux semer le désordre dans le récit, le politiser autour de la question du genre, et même jeter le trouble dans l'historiographie littéraire, en ramenant au centre de ses préoccupations les questions de hantise, de survivance, d'héritage, de tradition, de responsabilité, de dette et de justice. Comme la fable d'Oscar Wilde « The Fisherman and His Soul » et The Picture of Dorian Gray, ce roman de « Lucas Malet » (Mary St Leger Kingsley Harrison, 1852-1931) mêle la fascination fin-de-siècle pour les rites catholiques, l'occulte, le surnaturel, les portraits vivants, l'ésotérique et le spiritisme avec les ingrédients classiques du roman gothique, qui repose sur la dialectique de l'impalpable rendu visible.

The Gateless Barrier (1900) is a novel concerned with the visual as defined by Georges DidiHuberman, that is, with the inexplicable but compelling, spectral power of images. Focusing on the fate of the charming Agnes Rivers, whose ghost haunts the Rivers' ancestral house, The Gateless Barrier breaks with the realist tradition as well as with the aesthetic novel, in order to challenge contemporary gender politics and ultimately revise literary historiography. Part New Woman novel, part Gothic romance, the novel explores matters of haunting and the spectral and addresses issues of memory, responsibility, justice and debt. Not unlike Oscar Wilde's tale « The Fisherman and His Soul " or The Picture of Dorian Gray, this novel by «Lucas Malet " (Mary St Leger Kingsley Harrison, 1852-1931) combines a fin-de-siècle fascination for Catholic rituals, the occult, the supernatural, living portraits, esoterism, and spiritism with the classic ingredients of the Gothic novel, in order to examine the dialectics of the visible and the invisible.

\section{INDEX}

oeuvrecitee Gateless Barrier (The)

Keywords : gothic, aestheticism, decadence, feminism, literary history, haunting, ethics, visual, image

Mots-clés : gothique, esthétisme, décadence, féminisme, histoire de la littérature, hantise, éthique, visuel, image

\section{AUTEURS}

\section{CATHERINE DELYFER}

Catherine Delyfer est Professeur des Universités à l'Université Toulouse Jean Jaurès et codirectrice de la revue Cahiers victoriens et édouardiens. Elle s'intéresse à l'art, la littérature et la civilisation de la fin de l'époque victorienne, notamment au rapport texte/image et aux questions 
de genre/gender. Elle vient de publier une monographie intitulée Art and Womanhood in Fin-deSiècle Writing: the Fiction of Lucas Malet (1880-1931) (Londres: Pickering and Chatto, 2011). 